Working Paper 93-39

Statistics and Econometrics Series 25

November, 1993
Departamento de Estadística y Econometría Universidad Carlos III de Madrid

Calle Madrid, 126

28903 Getafe (Spain)

Fax (341) 624-9849

\title{
DIAGNOSTICS AND ROBUST ESTIMATION IN MULTIVARIATE DATA TRANSFORMATIONS
}

Santiago Velilla*

Abstract

This paper presents a method for detecting multivariate outliers which might be distorting the estimation of a transformation to normality. A robust estimator of the transformation parameter is also proposed.

Key Words

Likelihood displacement, multivariate Box-Cox transformation, $\mathbf{S}$ estimators.

•Departamento de Estadística y Econometría, Universidad Carlos III de Madrid. 


\section{DIAGNOSTICS AND ROBUST ESTIMATION IN MULTIVARIATE DATA TRANSFORMATIONS}

Santiago Velilla, Dpta. de Estadistica \& Econametria, Universidad Carlas III de Madrid, 28903-Setafe (Madrid), Ypain.

Alestract: This paper presents a method for detecting multivariate outliers which might be distorting the estimation of a transformation to normality. A robust estimator of the transformation parameter is also proposed.

Keqwards and phrases: Likelihood displacement; Multivariate Box-Cox transformation; S estimators.

\section{Introduction}

Consider a $\mathrm{p}$-variate random vector $\mathrm{X}=\left(\mathrm{X}_{1}, \ldots, \mathrm{X}_{\mathrm{p}}\right)^{\prime}$ such that all its components take positive values. If $\mathbf{X}$ is not multivariate normal, Andrews et al. (1971) propose the following transformation method to normality. Defining for a>0 and scalar $\lambda$ the family of transformations

$$
a^{(\lambda)}= \begin{cases}\frac{a^{\lambda}-1}{\lambda}, & \lambda \neq 0 ; \\ \log a, & \lambda=0,\end{cases}
$$

they consider a vector $\Lambda=\left(\lambda_{1}, \ldots, \lambda_{p}\right)^{\prime}$ of transformation parameters, one for each dimension, such that when transforming each $x_{j}$ in the form $x_{j}\left(\lambda_{j}\right)$, the following model holds, at least approximately,

$$
\mathrm{x}^{(\Lambda)}=\left(\mathrm{x}_{1}^{\left(\lambda_{1}\right)}, \ldots, \mathrm{x}_{\mathrm{p}}^{\left.\left(\lambda_{\mathrm{p}}\right)^{\prime}\right) \sim \mathrm{N}_{\mathrm{p}}(\mu, \Sigma)}\right.
$$

where $\mu=\left(\mu_{1}, \ldots, \mu_{p}\right)^{\prime}$ and $\Sigma=\left(\sigma_{1 j_{\text {pxp }}}{ }^{\cdot}\right.$ Model (1.2) is a multivariate generalization of the univariate transformation model for a random variable $X$ of Box and Cox (1964), namely, $x^{(\lambda)} \sim N\left(m, \sigma^{2}\right)$,

While extensively studied both in the one sample case and in the multiple linear regression case (see e.g. Atkinson (1985)), the application of the Box-Cox transformation to multivariate data has received little attention in the literature. Since model (1.2) implies the $p$ marginal models $x_{j}^{(\lambda)} \sim N\left(\mu_{j}, \sigma_{j j}\right), \quad j=1, \ldots, p, \quad$ it is usually recommended, on the basis of numerical simplicity, to estimate $\Lambda$ by $\hat{\lambda}_{M}=\left(\hat{\lambda}_{1 M}, \ldots, \hat{\lambda}_{\mathrm{pM}}\right)^{\prime}$, where $\hat{\lambda}_{\mathrm{JM}}$ is the maximum likelihood estimator (MLE) of $\lambda_{j}$ computed under the $j$ th marginal model. It is argued that, in general, these marginal estimators will not differ from the MLE $\hat{\Lambda}$ of $\Lambda$ computed from the joint model (1.2) and, as a consequence, ..there is a . common belief that the problems encountered in 
dealing with multivariate data transformations can be handled by just using routine extensions of univariate techniques. On the other hand, it is well known that the MLE estimator to normality is very sensitive to outlying observations and as, a consequence, there is the need of developping both diagnostic techniques and robust estimation procedures for the multivariate transformation parameter $\Lambda$. In agreement with the ideas above, both univariate diagnostic techniques and robust estimation applied separately to each parameter $\lambda$, would provide, in principle, a reasonably satisfactory joint methodology for detecting and/or accommodating anomalous observations.

The aim of this paper is to propose specific multivariate diagnostic methods and rooust estimation procedures which are shown to be, either theoretically or by example, superior to simultaneous application of existing univariate techniques. Section 2 presents some background and motivation. Section 3 is devoted to diagnostics while section 4 is devoted to robustness. Section 5 contains some final comments.

\section{Background and motivation}

Let $x=\left(x_{1 j}\right)=\left(x_{1}, \ldots, x_{p}\right)$ be a $n \times p$ data matrix from a random vector $\mathrm{X}$ with unkrown distribution F. If, according to the model (1.2), the rows of the transformed data matrix $x^{(\Lambda)}$, namely, $x_{1}^{(\Lambda)}=\left(x_{11}^{\left(\lambda_{11}\right)}, \ldots, x_{i p}^{\left(\lambda_{i p}\right)}\right), i=1$, $\ldots, n$, are i.i.d. $\mathrm{N}_{\mathrm{p}}(\mu, \Sigma)$, it can be shown that the concentrated $\log$-likelihood $\mathrm{L}_{\max }(\Lambda)$ for $\Lambda$ is (up to an additive constant)

$$
\left.\mathrm{L}_{\max }(\Lambda)=-\frac{\mathrm{n}}{2} \log || \mathrm{Z}^{(\Lambda)^{\prime}} \mathrm{AZ} \mathrm{Z}^{(\Lambda)} \mid\right] \text {, }
$$

where $z^{(\Lambda)}=\left(z_{i j}\left(\lambda_{j}\right)=\left(z_{1}^{\left(\lambda_{1}\right)}, \ldots, z_{p}^{\left(\lambda_{p}\right)}\right)\right.$ is the $n \times p$ matrix of normalized

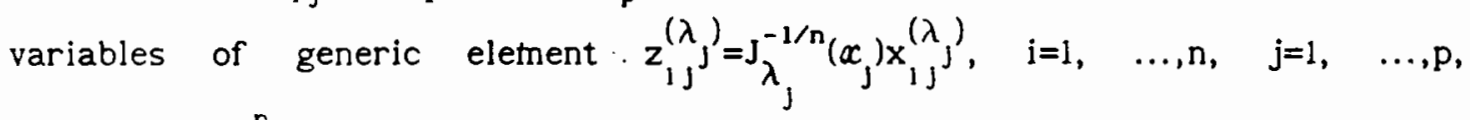
and $J_{\lambda_{j}}\left(x_{j}\right)=\left(\prod_{1=1}^{n} x_{1 j}\right)^{\lambda},-1, j=1, \ldots, p$, are the jacobian terms. The matrix $A$ in (2.1) is the $n \times n$ projection matrix $A=I_{n}-11_{n}^{1} / n$. The $\operatorname{MLE} \hat{\Lambda}=\left(\hat{\lambda}_{1}, \ldots, \hat{\lambda}_{p}\right)$ minimizes then the determinant

$$
\left|z^{(\Lambda)^{\prime}} A z^{(\Lambda)}\right|
$$

However, as mentioned in the introduction, the customary practice is to estimate $\Lambda$ by the estimator $\hat{\lambda}_{\mathrm{M}}=\left(\hat{\lambda}_{1 \mathrm{M}}, \ldots, \hat{\lambda}_{\mathrm{pM}}\right)^{\prime}$. It can be shown by induction (see appendix $A$ ) the identity

$$
\left|Z^{(\Lambda)^{\prime}} A Z^{(\Lambda)}\right|=\left[\prod_{k=1}^{p} Z_{k}^{(\lambda){ }^{\prime}} A Z_{k}^{(\lambda)}\right]_{k=1}^{p} \prod_{k}^{\left(1-r_{k}^{2}\right)}
$$

where,$r_{1}^{2}=0$ and, for $k \geq 2 ; \cdot r_{k}^{2}=r_{k}^{2}\left(\lambda_{1}, \ldots, \lambda_{i k}\right)$ is the multiple correlation : 
coefficient of $z_{k}^{\left(\lambda_{k}\right)}$ with $\left(z_{1}^{\left(\lambda_{1}\right)}, z_{k-1}^{\left(\lambda_{k-1}\right)}\right)$. (2.3) decomposes the objective function for $\hat{\Lambda}$ in the product of the $p$ marginal objective functions plus a factor which depends on the sequence $\left\langle r_{k}^{2}\right\}$. In applications, $\left\langle r_{k}^{2}\right\}$ is, in general, quite stable for all the values of the transformation parameter $\Lambda$ in a neighborhood of the optimum $\hat{\Lambda}$ and therefore, the relevant information in the determinant criterion $\left|\mathbf{Z}^{(\Lambda)^{\prime}} A Z^{(\Lambda)}\right|$ comes from the marginal criterions

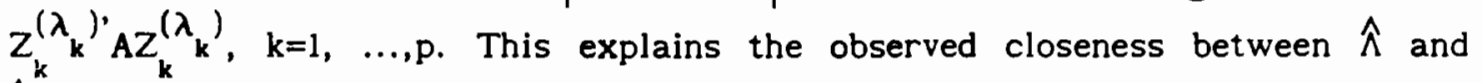
$\hat{\Lambda}_{\mathrm{M}}$. This empirical phenomenon is further illustrated in examples 2.1 and 2.2 below. In experience of the author, the function $-L_{\max }(\Lambda)$ is typically convex, a convenient feature for numerical optimization using a canned routine. Exact expressions for the partial derivatives in the gradient vector $\partial \mathrm{L}_{\max }(\Lambda) / \partial \Lambda$ and the Hessian matrix $\partial\left(\partial \mathrm{L}_{\max }(\Lambda) / \partial \Lambda\right)^{\prime} / \partial \Lambda$ of $\mathrm{L}_{\max }(\Lambda)$ are in appendix $B$.

EXAMPLE 2.1. A random sample of size $n=50$ is generated trough the model

$$
(\log X, \log Y)^{\prime} \sim \mathrm{N}_{2}\left[\left(\begin{array}{l}
0 \\
0
\end{array}\right),\left(\begin{array}{cc}
1 . & .95 \\
.95 & 1 .
\end{array}\right)\right]
$$

By applying a standard Newton-Raphson algorithm in each coordinate, we get $\hat{\Lambda}_{M}=(-.0441,-.1474)^{\prime}$. Using $\hat{\Lambda}_{M}$ as the initial point in the corresponding bivariate optimization, we find $\hat{\Lambda}=(-.0594,-.1812)$. The function $1-r_{2}^{2}\left(\lambda_{1}, \lambda_{2}\right)$ varies between .1159 and .1214 in the rectangle $[-.1,0] \times.[-.2,-.1]$.

EXAMPLE 2.2. A random sample of size $n=50$ is generated trough the trivariate lognormal model

$$
(\log X, \log Y, \log Z)^{\prime} \sim N_{3}\left[\left(\begin{array}{l}
0 \\
0 \\
0
\end{array}\right) \cdot\left(\begin{array}{ccc}
1 . & .75 & .6 \\
.75 & 1 . & .5 \\
.6 & .5 & 1 .
\end{array}\right)\right]
$$

Using the same methodology as above, we find $\hat{\Lambda}_{M}=(.0354,-.1354,-.1740)^{\prime}$ and $\hat{\Lambda}=(.0443,-.1467,-.1543)^{\prime}$. In $\because$ the rectangle $[0 ., 11] \times[-.2,-.1]_{x}[-.2,-.1]$, the function $1-r_{2}^{2}\left(\lambda_{1}, \lambda_{2}\right)$ is bounded between .431 and .436 and $1-r_{2}^{2}\left(\lambda_{1}, \lambda_{2}, \lambda_{3}\right)$ between .642 and .662 .

\section{Diagnostics}

We will be interested in constructing a diagnostic for the effect of single case deletion on $\hat{\Lambda}$. The notation $\hat{\Lambda}_{(1)}$ will be used for the estimator computed after deletion of the ith row of the data matrix. For the univariate case, case deletion diagnostics have been' proposed by cook and. 
Wang (1983), Hinkley and Wang (1988) and Tsai and Wu (1990). Specifically, Cook and Wang (1983) proposed taking the likelihood displacement

$$
L D_{1}=2\left[L_{\max }(\hat{\lambda})-L_{\max }\left(\hat{\lambda}_{(1)}\right)\right] \text {, }
$$

as a scalar measure of influence. Cook and Wang (1983) suggested also a numerical approximation for the perturbed estimators $\hat{\lambda}_{(1)}, i=1, \ldots, n$, which is improved by Tsai and wu (1990). The following example shows how, for $p>1$, an outlier can remain undetected when applying the univariate diagnostic techniques separately in each one of the coordinates.

EXAMPLE 3.1. An cutlier $\left(x_{51}, y_{51}\right)^{\prime}$ is added to the data set in example 2.1 in such a way that $\left(\log x_{51}, \log \psi_{51}\right)=(2.1,-1.9)^{\prime}$. Plotting the data in logs, figure 3.1, it is easily seen that $\left(\log x_{51}, \log y_{51}\right)$ alters the structure of correlation of the cloud defined by the bulk of the remaining 50 data points. Now we get $\hat{\Lambda}_{M}=(-.0612,-.0928)^{\prime}$ and $\hat{\Lambda}=(-.1393, .0252)^{\prime}$. Figure 3.2 a) and figure 3.2 b) show, for $j=1,2$, the marginal diagnostics

and

$$
\hat{\lambda}_{j M(j)}^{1}
$$

$$
L D_{!}^{J}=2\left[L_{\max }^{J}\left(\hat{\lambda}_{j M}\right)-L_{\max }\left(\hat{\lambda}_{j M(i)}^{1}\right)\right],
$$

respectively, where $\hat{\lambda}_{j M}^{1}$ is the corresponding marginal one-step approximation of Tsai and Wu (1990). Figure $3.2 \mathrm{cl}$ displays the combined marginal diagnostic $L D_{i}^{1}+L D_{i}^{2}$. Notice that case 51 remains unnoticed.

Figure 3.1

Figure 3.2 a) Figure 3.2 b) Figure $3.2 \mathrm{cl}$

In the example above, marginal diagnostics fail because they don't take into account the correlation, structure of the transformed data. In principle, a suitable multivariate diagnostic measure would be

$$
L D_{1}=2\left[L_{\max }(\hat{\Lambda})-L_{\max }\left(\hat{\Lambda}_{(1)}\right)\right]
$$

By (2.1), the measure (3.2) has a cumbersome expression and is hard to work with. By a standard first order Taylor expansion and using that $\partial \mathrm{L}_{\max }(\Lambda) /\left.\partial \Lambda\right|_{\Lambda=\hat{\Lambda}}=0$, we can approximate

$$
\angle D_{1} \cong \tilde{L} D_{1}=\left(\hat{\Lambda}-\hat{\Lambda}_{(1)}\right)^{\prime} H(\hat{\Lambda})\left(\hat{\Lambda}-\hat{\Lambda}_{(i)}\right)
$$


where $H(\hat{\Lambda})=-\left.\frac{\partial}{\partial \Lambda}\left(\frac{\partial}{\partial \Lambda} L_{\max }(\Lambda)\right)^{\prime}\right|_{\Lambda=\hat{\Lambda}}$ is the pxp observed Fisher information matrix. Velilla (1993) proposes the ellipsoid

$$
(\Lambda-\hat{\Lambda})^{\prime} H(\hat{\Lambda})(\Lambda-\hat{\Lambda}) \leq \chi_{p, \alpha^{\prime}}^{2}
$$

as an alternative asymptotic $(1-\alpha) \times 100 \%$ confidence region for the transformation parameter. Therefore, calibration of the influence measure $\tilde{\mathrm{L}} \mathrm{D}_{1}$ on the right hand side of (3.3) can be made with reference to the percentage points of the $\chi_{p}^{2}$ distribution.

From the computational point of view, $\tilde{L} D_{i}$ depends on: (i) The MLE $\hat{\Lambda}$; (ii) The matrix $H(\hat{\Lambda})$; and (iii) The estimators $\hat{\Lambda}_{(i)}, i=1, \ldots, n$. The MLE is obtained by maximizing $L_{\max }(\Lambda)$ and the elements of the matrix $H(\hat{\Lambda})$ can be computed from the corresponding expressions in theorem B.1 of appendix $B$, with the additional simplification obtained from $\partial L_{\max }(\Lambda) /\left.\partial \lambda_{r}\right|_{\Lambda=\hat{\Lambda}}=0$. Computation of $\hat{\Lambda}_{(1)}$ requires iteration and an approximation is developed as follows. By (2.2) the MLE $\hat{\Lambda}$ minimizes $|M(\Lambda)|$, where $M(\Lambda)=\left|Z^{(\Lambda)^{\prime}} A Z^{(\Lambda)}\right|$, and, therefore, $\hat{\Lambda}_{(1)}$ minimizes, in obvious notation, $\left|M_{(i)}(\Lambda)\right|$. Let $\nabla_{(1)}(\Lambda)=\partial\left|M_{(1)}(\Lambda)\right| / \partial \Lambda$ and $H_{(i)}(\Lambda)=$ $\partial^{2}\left|M_{(i)}(\Lambda)\right| \cdot \partial \Lambda \partial \Lambda^{\prime}$ be, respectively, the gradient vector and the hessian matrix of $\left|M_{(i)}(\Lambda)\right|$. In the first-order Taylor expansion around an initial guess $\Lambda_{0}$ for $\hat{\Lambda}_{(i)}, \nabla_{(i)}(\Lambda) \cong \nabla_{(i)}\left(\Lambda_{0}\right)+H_{(1)}\left(\Lambda_{0}\right)\left(\Lambda-\Lambda_{0}\right)$, we can use the fact that $\nabla_{(i)}\left(\hat{\Lambda}_{(i)}\right)=0$ to obtain a one-step approximation for $\hat{\Lambda}_{(i)}$,

$$
\hat{\Lambda}_{(1)}^{1}=\Lambda_{0}-\left[H_{(1)}\left(\Lambda_{0}\right)\right]^{-1} \nabla_{(1)}\left(\Lambda_{0}\right)
$$

Equation (3.5) is a multivariate extension of equation (15) in Tsai and Wu (1990). Typically $\Lambda_{0}=\hat{\Lambda}$ and the computational problem is finished whenever we are able to compute the explicit expressions for the elements in $\nabla_{(1)}(\hat{\Lambda})$ and $H_{(i)}(\hat{\Lambda})$. These can be seen in appendix $B$.

EXAMPLE 3.1 (cont.). Figure 3.3 a) is an index plot of $L D_{1}$ where case 51 is clearly pinpointed. Figure 3.3 b) is the corresponding plot for $\tilde{L D}_{i}$. Deletion of case 51 moves the estimator to the boundary of a 99.25\% confidence ellipsoid.

\section{Figure 3.3 a)}

Figure 3.3 b)

Finally, it is important to remark that in both (3.3) and (3.4) it is crucial to use the multivariate estimators $\hat{\Lambda}_{\text {. and }} \hat{\lambda}_{(1)}$. By. ,replacing, in (3.3). $\hat{\Lambda}$ and $\hat{\Lambda}_{(1)}$ by their marginal counterparts $\hat{\Lambda}_{M}$ and $\cdot \hat{\Lambda}_{M(1)}$ the : 
"marginal" version of (3.3), namely $L D M_{1}=2\left[L_{\max }\left(\hat{\Lambda}_{M}\right)-L_{\max }\left(\hat{\Lambda}_{M(1)}\right)\right]$ of ten takes negative values. On the other hand, ${\tilde{L} D M_{1}}_{1}\left(\hat{\Lambda}_{M}-\hat{\Lambda}_{M(1)}\right)^{\prime} H\left(\hat{\Lambda}_{M}\right)\left(\hat{\Lambda}_{M}-\hat{\Lambda}_{M(1)}\right)^{\prime}$ does not indicate the outlier because of the lack of consideration of the correlation structure. See figures 3.4 a) and b). Moreover, the general claim of closeness between $\hat{\lambda}$ and $\hat{\lambda}_{M}$ is not true in the presence of outliers. If, for a given $p \times 1$ vector $u,\|u\|_{M}^{2}=u^{\prime} M u$ denotes the square of the norm of $u$ with respect the inner product pxp matrix $M$, we have, in the case of example 3.1, $\left\|\hat{\Lambda}-\hat{\Lambda}_{M}\right\|_{H}^{2}=2.06$ and $\left\|\hat{\Lambda}_{(51)}-\hat{\Lambda}_{M(51)}\right\|_{H}^{2}=.08$.

Flgure 3.4 a)

Flgure 3.4 b)

\section{A robust estimator of the transformation parameter}

As a consequence of section 3, a multivariate outlier can remain undetected if only univariate diagnostic techniques are used. Therefore, if a robust estimator of the transformation parameter $\Lambda=\left(\lambda_{1}, \ldots, \lambda_{p}\right)^{\prime}$ is to be constructed, it should not be based in robustifying separately in each dimension. This section presents a robust estimator $\hat{\lambda}_{R}$ of $\Lambda$ which takes into account the correlation structure among the transformed variables.

Given $x_{1}, \ldots, x_{n}$ i.i.d. p-variate observations with unknown distribution $F$ that can be approximately modelled with the transformation model (1.1), the corresponding log-likelihood is (up to an additive constant)

$$
L(\mu, \Sigma, \Lambda)=-(n / 2) \log |\Sigma|-(1 / 2) \sum_{i=1}^{n}\left(x_{1}^{(\Lambda)}-\mu\right)^{\prime} \Sigma^{-1}\left(x_{1}^{(\Lambda)}-\mu\right)+\log \left[J_{\Lambda}(X)\right],
$$

where $\mathrm{J}_{\Lambda}(x)=\prod_{j=1}^{\mathrm{F}} \mathrm{J}_{\lambda}\left(x_{j}\right), \quad \mathrm{J}_{\lambda_{j}}\left(x_{j}\right)=\left(\prod_{i=1}^{n} x_{1 j}\right)^{\lambda}{ }_{j}^{-1}$, is the jacobian term. The MLE estimator $(\hat{\mu}, \hat{\Sigma}, \hat{\Lambda})$ is obtained maximizing in $\Lambda$ the profile log-likelihood $L_{\max }(\Lambda)=L(\hat{\mu}(\Lambda), \hat{\Sigma}(\Lambda), \Lambda)$, with $\hat{\mu}(\Lambda)=n^{-1} \sum_{1=1}^{n} x_{1}^{(\Lambda)}, \quad \hat{\Sigma}(\Lambda)=n^{-1} \dot{x}^{(\Lambda)} A x^{(\Lambda)}$, which can be written in the form

$$
-(n / 2) \log [|\hat{\Sigma}(\Lambda)|]+\log \left[J_{\Lambda}(X)\right] .
$$

From (4.2), a possible method for robustifying the estimation of $\Lambda$ is to replace the objective function (4.2) by:

$$
L_{\max , R}(\Lambda)=-(n / 2) \log \left[\left|\sum_{R}(\Lambda)\right|\right]+\log \left[J_{\Lambda}(x)\right] .
$$

where $\sum_{R}(\Lambda)$ is a robust estimator of $\Sigma$ computed from the data matrix $x^{(\Lambda)}$. our choice for. $\sum_{R}\left(\Lambda^{\prime}\right)$ is the - S-estimator. of dispersion as described in . Lopuhaä (1989): For the univariate case, Carroll (1980) has proposed a 
robust estimation approach by replacing, in the normal log-likelihood for $(\mu, \sigma, \lambda), \quad d_{1}^{2} / 2=\left(x_{1}^{(\lambda)}-\mu\right)^{2} / 2 \sigma^{2}$ by $\rho\left(d_{1}\right)$. For every fixed $\lambda$, he suggests estimating $\mu$ and $\sigma$ for $M$-estimators $\mu_{M}(\lambda)$ and $\sigma_{M}(\lambda)$ and to estimate $\lambda$ by maximizing the function $-(n / 2) \log \left[\sigma_{M}^{2}(\lambda)\right]-\sum_{l=1}^{n} \rho\left[\left(x_{1}^{(\lambda)}-\mu_{M}(\lambda)\right) / \sigma_{M}(\lambda)\right]+$ $(\lambda-1) \sum_{i=1}^{n} \log \left(x_{1}\right)$. For $p=1$, our proposal maximizes $-(n / 2) \log \left[\sigma_{s}^{2}(\lambda)\right]$ $\sum_{i=1}^{n} \rho\left[\left(x_{i}^{(\lambda)}-\mu_{s}(\lambda)\right) / \sigma_{s}(\lambda)\right]+(\lambda-1)+\sum_{i=1}^{n} \log \left(x_{1}\right)$. For the univariate case, other work in robustness in transformations can be seen in Carroll and Ruppert (1988).

Next we study the properties and discuss the computation of $\hat{\lambda}_{R}$. We also propose a measure of its robustness under contamination by analyzing the expression of its influence function. For the most part, the following discussion is heuristic and concentrates mainly on the ideas. More technical details can be found in appendix $C$.

\subsection{Existence of solutions, consistency and asymptotic normality}

Appendix $C$ contains a brief description on conditions which imply the existence, for all $n$ large enough, of a sequence of solutions $\left\{\hat{\Lambda}_{R}\right\}$ of (4.3) such that

$$
\hat{\Lambda}_{R} \longrightarrow \Lambda_{0} \text {, a.e, }
$$

being $\Lambda_{0}=\Lambda_{0}(F)$ the unique global maximum over $\Lambda \in \mathbb{K}$ of

$$
-(1 / 2) \log [|\Sigma(\Lambda, F)|]+\Lambda^{\prime} u
$$

where $k$ is a compact set of $\mathbb{R}^{F}, u=\left(E_{F}\left[\left|\log X_{i}\right|\right], \ldots, E_{\bar{F}}\left[\left|\log X_{p}\right|\right]\right)$ and $\Sigma(\Lambda, F)$ is the corresponding block of the solution $\theta(\Lambda, F)=(\mu(\Lambda, F), \Sigma(\Lambda, F))$ of the S-estimation problem $\min |\Sigma|$ over $\theta=(\mu, \Sigma) \quad(\Sigma>0)$ restricted to $\int \rho\left[\left\{\left(x^{(\Lambda)}-\mu\right)^{\prime} \Sigma^{-1}\left(x^{(\Lambda)}-\mu\right)\right\}^{1 / 2}\right] F(d x)=k_{p}$. In agreement with this notation, $\left(\hat{\mu}_{R}(\Lambda), \sum_{R}(\Lambda)\right)=\theta\left(\Lambda, F_{n}\right)$. We delete sometimes dependence on $F$ and simply write $\theta(\Lambda, F)=\theta(\Lambda)$.

Next we assume that $\Lambda_{0}$ is an interior point of $\mathbb{K}$ and write $\theta_{0}=\left(\mu_{0}, \Sigma_{0}\right)=\theta\left(\Lambda_{0}, F\right)$. By expression (2.7) in Lopuhaä (1989), for each $\Lambda$ in $K$, $\theta(\Lambda)$ solves

$$
H(\Lambda, \theta)=\int \psi(x, \Lambda, \theta) F(d x)=0,
$$

where the function $\psi=\left(\psi_{1}^{\prime}, \psi_{2}^{\prime}\right)$ has components:

$$
\psi_{1, r}(x, \Lambda ; \theta)=\dot{u}[d]\left(\dot{x}_{r}^{(\lambda)}{ }_{r}-\mu_{r}\right), r=1, \ldots, p ;
$$




$$
\psi_{2, r s}(x, \Lambda, \theta)=p u[d]\left(x_{r}\left(\lambda_{r}\right)-\mu_{r}\right)\left(x_{s}(\lambda)-\mu_{s}\right)-v[d] \sigma_{r s^{\prime}} \quad r, s=1, \quad \ldots, p, \quad(r \leq s),
$$

where $\quad d=d(x, \Lambda, \theta)=\left[\left(x^{(\Lambda)}-\mu\right)^{\prime} \Sigma^{-1}\left(x^{(\Lambda)}-\mu\right)\right]^{1 / 2}, \quad u[d]=\varphi[d] / d, \quad$ and $\quad v[d]=t \varphi[d]$ $-\rho[d]+k_{p}$, being $\varphi$ the derivative $\rho$ of $\rho$. By assuming an appropriate form for $\rho[$.$] and suitable conditions on the moments of F$, it can be shown that $H(\Lambda, \theta)$ has continuous second partial derivatives in a neighborhood of $\left(\Lambda_{0}, \theta_{0}\right)$ which can be obtained differentiating under the integral sign in (4.5). Since $H\left(\Lambda_{0}, \theta_{0}\right)=0$, if $\partial H(\Lambda, \theta) /\left.\partial \theta\right|_{(\Lambda, \theta)=\left(\Lambda_{0}, \theta_{0}\right)}$ is nonsingular, by the implicit function theorem (see, e.g. Fleming (1977, p.148)), there exists, locally around $\Lambda_{0}$, a function $\theta(\Lambda)$ with continuous second partial derivatives such that $H(\Lambda, \theta(\Lambda))=0$. We introduce now the set of artificial parameters $W=\left\{\left\{\omega_{r, j}\right\}, \quad\left\{\omega_{r s, j}\right\}, \quad\left\{\omega_{r, j k}\right\}, \quad\left\{\omega_{r s, j k}\right\}: \quad r, s=1, \ldots, p, \quad(r \leq s)\right.$, $\mathrm{j}, \mathrm{k}=1, \ldots, \mathrm{p}\rangle$, which have the meaning $\omega_{r, j}=\partial \mu_{r} / \partial \lambda_{j}, \omega_{r s, j}=\partial \sigma_{r s} / \partial \lambda_{j}$, $\omega_{r, j k}=\partial^{2} \mu_{r} / \partial \lambda_{j} \partial \lambda_{k}$, and $\omega_{r s, j k}=\partial^{2} \sigma_{r s} / \partial \lambda_{j} \partial \lambda_{k}$. The following equation holds

$$
\int \dot{\psi}(\mathrm{x}, \Lambda, \theta, W) F(d x)=0,
$$

where $\dot{\psi}$ is the array $\partial \psi / \partial \Lambda$. Moreover, $\Lambda_{0}$ satisfies

$$
\int \Gamma(\mathrm{x}, \Lambda, \theta) F(\mathrm{~d} x)=0,
$$

where $\Gamma=\left\{\Gamma_{j}\right\}$ is the pxl gradient vector of the function in (4.4). Explicit expressions for the components of $\dot{\psi}$ and $\Gamma$ are in appendix $D$. In summary, if $\Omega=(\Lambda, \theta, W), \Lambda_{0}$ is the corresponding component of the solution $\Omega_{0}$ of the equation

$$
\int \Phi(x, \Omega) F(d x)=0,
$$

where $\Phi(x, \Lambda, \theta)=(\Gamma, \psi, \dot{\psi})$. By replacing, in (4.9), $F$, by the empirical $F_{n}$, we get the estimating equation

$$
\mathrm{n}^{-1} \sum_{i=1}^{n} \Phi\left(x_{1}, \Omega\right) F(d x)=0,
$$

whose solution $\hat{\Omega}_{n}=\left(\hat{\Lambda}_{R}, \hat{\theta}_{n}, \hat{\omega}_{n}\right)$ determines $\hat{\Lambda}_{R}$. Observe that $\Omega$ and $\Phi$ are both of dimension $q=(p / 2)(2+(p+1)(p+3)]$. Equations (4.9) and (4.10) allow for an obtention of the asymptotic normality of $\hat{\Lambda}_{R}$ in the framework of M-estimation. By application of the conditions Huber (1967), if the function $\lambda_{F}[\Omega]=E_{F}[\Phi(x, \Omega)]$ has a nonsingular $q \times q$ derivative $D$ at $\Omega_{0^{\prime}}$ then $n^{1 / 2}\left(\Omega_{n}-\Omega_{0}\right)-\stackrel{D}{\longrightarrow} N_{q}\left[0, D^{-1} M\left(D^{-1}\right)^{\prime}\right]$, where $M=\operatorname{cov}_{F}[\Phi(x, \Omega)]$, and as a consequence,

$$
\mathrm{n}^{1 / 2}\left(\hat{\Lambda}_{\mathrm{R}}-\Lambda_{\mathrm{O}}\right) \stackrel{\mathrm{D}}{\longrightarrow} \mathrm{N}_{\mathrm{q}}^{\mathrm{F}}[0, \mathrm{HR}]
$$

where $H R$ is the proper pxp submatrix of $D^{-1} M\left(D^{-1}\right)$ '. A consistent estimate $H_{n}$ of $H R$ : is given by the associated pxp submatrix of $D_{n}^{-1} M_{n}\left(D_{n}^{-1}\right)$, where 
$D_{n}=n^{-1} \sum_{i=1}^{n} \partial \Phi\left(x_{1}, \Omega\right) /\left.\partial \Omega\right|_{\Omega=\Omega_{n}}$ and $M_{n}=n^{-1} \sum_{i=1}^{n} \Phi\left(x_{i}, A_{n}\right) \Phi\left(x_{1}, \Omega_{n}\right)^{\prime}$

\subsection{Computation of $\hat{\Lambda}_{R}$}

The numerical problem for computing $\hat{\Lambda}_{R}$ is to minimize in $\Lambda=\left(\lambda_{1}\right.$, $\left.\ldots, \lambda_{p}\right)^{\prime}$ the function

$$
h[\Lambda]=(1 / 2) \log \left[\left|\sum_{R}(\Lambda)\right|\right]-\sum_{1=1}^{n} \lambda_{j} p_{n, J}
$$

where $p_{n, j}=n^{-1} \sum_{i=1}^{n} \log \left(x_{i j}\right)$. The function used in the determination of the robust estimator $\sum_{R}(\Lambda)$ is the function

$$
\rho[t]= \begin{cases}\left(t^{6} / 6 c\right)-\left(t^{8} / 4 c^{3}\right)+\left(t^{10} / 10 c^{5}\right), & |t| \leq c ; \\ c^{5} / 60 & , \quad|t|>c\end{cases}
$$

for a suitable positive constant $c$. This function satisfies the regularity conditions of appendix $C$. Its derivative $\varphi[t]=\left(t^{5} / c\right)\left[1-(t / c)^{2}\right]^{2},|t| \leq c, \quad(0$ for $|t|>c)$ is a multiple of Tukey's biweight function. The first partial derivatives of $h[\Lambda]$ are

$$
\partial h / \partial \lambda_{j}=(1 / 2) \sum_{r, s=1}^{p}\left[2 \hat{\sigma}^{r s}-\delta_{r s} \hat{\sigma}^{r s s}\right] \hat{\omega}_{r s, j}-p_{n, j}
$$

$j=1, \ldots, p$. For every fixed $j$, the array $\left\{\hat{\omega}_{r s, j}\right\}$ is determined from the equation (4.7) or, more specifically, from the linear system

$$
A \hat{W}_{j}=\hat{b}_{j}
$$

where $\left.\hat{w}_{j}=\left\{\hat{\omega}_{r, j}\right\}, \quad\left\{\hat{\omega}_{r s, j}\right\}\right\}$ and the $[p(p+3) / 2]_{x} 1$ vector $\hat{b}_{j}=\left\{\hat{b}_{r, j} ; \hat{b}_{r s, j}\right\}$ and $[p(p+3) / 2] \times[p(p+3) / 2]$ matrix

$$
A=\left(\begin{array}{c|c}
\hat{\alpha}_{r, a} & \hat{\alpha}_{r, a b} \\
\hline \hat{\alpha}_{r s, a b} & \hat{\alpha}_{r s, a b}
\end{array}\right), \quad \quad \quad \quad \quad \quad
$$

have elements

$$
\begin{gathered}
\hat{b}_{r, j}=-n^{-1} \sum_{i=1}^{n} \partial \psi_{1, r} / \partial \lambda_{j}, \hat{b}_{r s, j}=-n^{-1} \sum_{i=1}^{n} \partial \psi_{2, r s} / \partial \lambda_{j}, \\
\hat{\alpha}_{r, a}=n^{-1} \sum_{i=1}^{n} \partial \psi_{1, r} / \partial \mu_{a}, \therefore \hat{\alpha}_{r, a b}=n^{-1} \sum_{i=1}^{n} \partial \psi_{1, r} / \partial \sigma_{a b} \\
\hat{\alpha}_{r s, a}=n^{-1} \sum_{i=1}^{n} \partial \psi_{i, r s} / \partial \mu_{a} \text { and } \hat{\alpha}_{r s, a b}=n^{-1} \sum_{1=1}^{n} \partial \psi_{1, r s} / \partial \sigma_{a b},
\end{gathered}
$$

for $r, s, a, b=1, \quad \ldots, p(r \leq s, a \leq b) . \quad$ All the derivatives are evaluated at 
$\left(\Lambda, \hat{\theta}_{R}(\Lambda)\right)$, where $\hat{\theta}_{R}(\Lambda)=\left(\hat{\mu}_{R}(\Lambda), \hat{E}_{R}(\Lambda)\right)$. Exact expressions can be found in appendix $D$. Recall that the matrix $A$ above is the same for every $j$. Second partial derivatives for $h$ theoretically exist but have untractable expressions. An algorithm for computing $\hat{\Lambda}_{R}$ based on a Newton-Raphson iteration which uses both the gradient $G=\partial h / \partial \Lambda$ and the Hessian matrix $H=\partial(\partial h / \partial \Lambda)^{\prime} / \partial \Lambda$ of $h$ is thus not recommended. We suggest instead the following algorithm for computing $\hat{\Lambda}_{R}$ :

(i) Start with an initial value of $\Lambda, \Lambda_{0}$ say, and compute the robust S-estimators $\hat{\theta}_{R}\left(\Lambda_{0}\right)=\left(\hat{\mu}_{R}\left(\Lambda_{0}\right), \hat{\Sigma}_{R}\left(\Lambda_{0}\right)\right)$ from the transformed data matrix $x^{\left(\Lambda_{0}\right)}$. If there is some previous diagnostic information, $\Lambda_{0}$ could be the approximation $\hat{\Lambda}_{(1)}^{1}$ computed deleting a dubious case $i$. A suitable algorithm for computing $\hat{\theta}_{R}\left(\Lambda_{0}\right)$ is given in Ruppert (1992).

(ii) Solve the collection of systems $A_{0} \hat{W}_{j 0}=\hat{b}_{j 0}, j=1, \ldots, p$, obtain the gradient $G_{0}$ of $h$ at $\Lambda=\Lambda_{0}$ and choose an initial guess for $H_{0}$, typically $\mathrm{H}_{0}=\mathrm{I}$.

(iii) Update $\left(\Lambda_{0}, G_{0}, H_{0}\right)$ to $\left(\Lambda_{1}, G_{1}, H_{1}\right)$ as in a Quasi-Newton algorithm with the BFGS formula (see, e.g. Seber and Wild (1989, pp. 605-609 for a description).

(iv) Iterate $(i)-(i i)-(i i i)$ until convergence.

\subsection{Influence function of $\hat{\Lambda}_{R}$}

The influence function of the functional $\Lambda_{0}=\Lambda(F)$ evaluated at a point $x \in \mathbb{R}^{p}$ and at underlying distribution $F$ is defined pointwise as the limit

$$
\lim _{h \rightarrow 0^{+}} \frac{\Lambda\left[(1-h) F+h \delta_{x}\right]-\Lambda[F]}{h},
$$

if the limit exists. If the conditions for existence and consistency hold and $\lambda_{F}[\Omega]=E_{F}[\Phi(x, \Omega)]$ has $a$ nonsingular $q \times q$ derivative $D$ at $\Omega_{0}$, then for the "larger" functional. $\Omega_{0}=\Omega(F)$, the influence function $\operatorname{IF}(x ; \Omega ; F)$ is the $q \times 1$ vector $-D^{-1} \Phi[x ; \Omega(F)]$. We have the sampling approximation $\operatorname{IF}(x ; \Omega ; F) \cong I \hat{F}_{n}=-D_{n}^{-1} \Phi\left[x ; \Omega_{n}\right]$. The influence function $\operatorname{IF}(x ; \Lambda ; F)$ is given by the first $p$ coordinates of $\operatorname{IF}(x ; \Omega ; F)$ with sampling approximation provided by the first $p$ coordinates of $I \hat{F}$.

\subsection{Examples}

EXAMPLE 4.1. We apply the algorithm described in 4.2 to the data set of example 3.1. We choose the constant $c=3$ in the definition of $\rho$ in (4.12). By. starting with $\Lambda_{0}=\hat{\Lambda}_{(51)}^{1}=(.0547,-.2024)^{\prime}$ and $\dot{H}_{0}=I_{2}$, we get $\hat{\Lambda}_{R}=(-.1057,-.2320)^{\prime}$ '. 
after three iterations. To assess the distance between $\hat{\Lambda}$ and $\hat{\Lambda}_{R}$. we see that $\left\|\hat{\Lambda}-\hat{\Lambda}_{R}\right\|_{M}^{2}=6.3876$, that is $\hat{\Lambda}_{R}$ lies autside the $95 \%$ confidence region (3.4). By comparison $\left\|\hat{\Lambda}_{(51)}-\hat{\Lambda}_{R}\right\|_{M}^{2}=.2919$.

EXAMPLE 4.2. Let $\hat{u}_{R}(x)$ the $p x 1$ vector formed by the first $p$ coordinates of the sampling approximation $\operatorname{IF}(x ; \Omega ; F) \cong] F_{n}=-D_{n}^{-1} \Phi\left[x ; \Omega_{n}\right]$. A suitable norming matrix for $\hat{u}_{R}(x)$ is the pxp matrix $H R$ introduced in 4.1

$$
\operatorname{IR}(x)=\left[\hat{u}_{R}(x)^{\prime}\left(H_{n} / n\right)^{-1} \hat{u}_{R}(x)\right]^{1 / 2} \text {. }
$$

The MLE estimator is also a particular case of M-estimator by choosing $u[d]=v[d]=1$ in (4.7). The influence function of the MLE can be found and, accordingly,

$$
\operatorname{IL}(x)=\left[\hat{u}_{L}(x)^{\prime}\left(H L_{n} / n\right)^{-1} \hat{u}_{L}(x)\right]^{1 / 2},
$$

can be computed. For the particular case of the data set in example 3.1, the vector $x=\left(x_{1}, x_{2}\right)$ ' has two coordinates, so we can transform to polar coordinates $x_{1}=r \cos \theta, \quad x_{2}=r \sin \theta \quad(r>0,0<\theta<\pi / 2)$ and compare the qualitative behaviour of $I R(x)$ and $\operatorname{IL}(x)$ with the two auxiliary curves $A R(r)=\sup I R(x)$ $A L(r)=\sup _{\|x\|=r} I L(x)$, where $\|x\|=\left[x_{1}^{2}+x_{2}^{2}\right]^{1 / 2}$. These appear in figure 4.1 , where

superiority of $\hat{\lambda}_{R}$ over $\hat{\lambda}$ is apparent.

Figure 4.1

\section{Final comments}

This paper presents methods of diagnostic and robustness for the transiormation parameter $\Lambda$ with multivariate data. Techniques presented are shown to be better than simultaneous application of previously suggested univariate methods. Computational issues are discussed. The ideas on robustness presented could be. in principle, adapted easily to the multiple regression case. The results of this paper would be an improvement over previous robust methods in regression because they present a procedure to compute a sampling approximation to the influence curve of the estimator.

\section{APPENDIX}

A Expression for the determinant (2.3). The result obviously holds for $p=2$. Asssuming it true for $p$, (2.3) follows from the well-known formula of the determinant of a partitioned squared matrix and induction hypothesis. . 
B Eradient and Hessian of $\mathrm{L}_{\max }(\Lambda)$. Theorem B.l. Introduce the notation: (i)

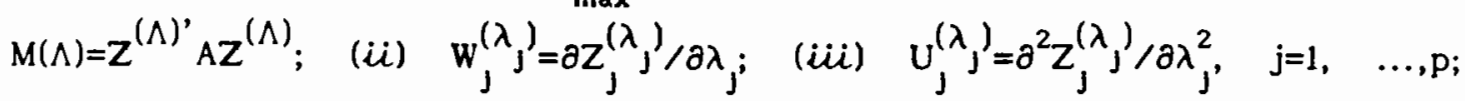
and (iv) For a nxp matrix $H=\left(h_{1}, \ldots, h_{p}\right), H_{j}(u)$ is $H$ with its $j$ th column replaced by the $n \times l$ vector $u$ and $H_{j k}(u, v)$ is $H$ with its $j$ th and kth column replaced by, respectively, $u$ and $v$. We have:

a) $\partial L_{\max }(\Lambda) / \partial \lambda_{r}=-n|M(\Lambda)|^{-1}\left|Z_{r}^{(\Lambda)}\left[W_{r}^{\left(\lambda_{r}\right)}\right] A Z^{(\Lambda)}\right|, r=1, \ldots p$;

b) $\partial^{2} L_{\max }(\Lambda) / \partial \lambda_{r}^{2}=$

$$
\begin{aligned}
-\Omega|M(\Lambda)|^{-2}\left[|M(\Lambda)|\left(\left|Z_{r}^{(\Lambda))^{\prime}}\left[U_{r}^{\left(\lambda_{r}\right)}\right] A Z^{(\Lambda)}\right|+\left|Z_{r}^{(\Lambda)^{\prime}}\left[W_{r}^{\left(\lambda_{r}\right)}\right] A Z_{r}^{(\Lambda)}\left[W_{r}^{\left(\lambda_{r}\right)}\right]\right|\right)\right. \\
\left.+\left|Z_{r}^{(\Lambda)^{\prime}}\left[W_{r}^{\left(\lambda_{r}\right)}\right] A Z^{(\Lambda)}\right|^{2}\right], r=1, \ldots, p ;
\end{aligned}
$$

c) $\partial^{2} L_{\max }(\Lambda) / \partial \lambda_{s} \partial \lambda_{r}=$

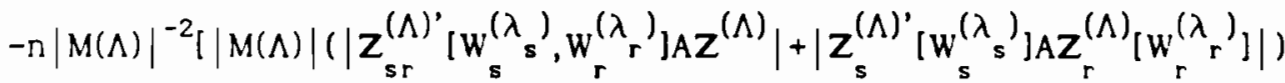

$$
\begin{aligned}
& \left.+\left|Z_{s}^{(\Lambda)^{\prime}}\left[W_{s}^{\left(\lambda_{s}\right)}\right] A Z^{(\Lambda)}\right|\left|Z_{r}^{(\Lambda)^{\prime}}\left[W_{r}^{\left(\lambda_{r}\right)^{\prime}}\right] A Z^{(\Lambda)}\right|\right] \text {, }
\end{aligned}
$$

for $r, s=1, \ldots, p(r \neq s)$.

B. 1 follows Velilla (1993). Now define, for $i=1, \ldots, n, j=1, \ldots, p$ : (i) The array of constants $a_{i j}=x_{i j}^{1 / n}\left(\prod_{k \neq i}^{n} x_{k j}\right)^{-1 / n(n-1)}$ and the furictions $q_{i j}\left(\lambda_{j}\right)=a_{i j}^{\lambda} j^{-1}$; (ii) The nxp matrix $z_{i}^{(\Lambda)}=\left(z_{i 1}^{\left(\lambda_{1}\right)}, \ldots, z_{i p}^{\left(\lambda_{p}\right)}\right)$ of jth column $Z_{i j}^{\left(\lambda_{j}\right)}=q_{i j}\left(\lambda_{j}\right) Z_{j}^{\left(\lambda_{j}\right)}$; and (iii) The $n \times n$ matrix $A_{i}=A_{i} A_{\text {, }}$ where $B_{i}=I-[1-(1 / n)]^{-1} e_{i} e_{i}^{\prime}$ and $e_{i}$ is the ith canonical vector of $\mathbb{R}^{n}$.

Theorem B.2. Define, for $i=1, \ldots, n$ and $j=1, \ldots, p$, the $n \times l$ vectors

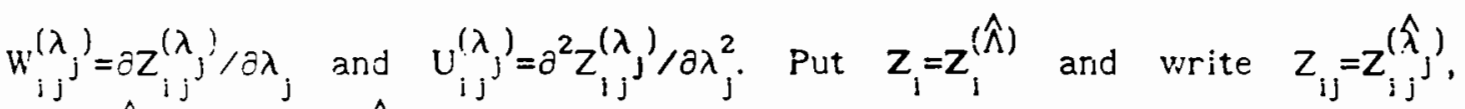
$w_{i j}=w_{i j}^{\left(\hat{\lambda}_{j}\right)}$, and $U_{i j}=U_{i j}^{\left(\hat{\lambda}_{j}\right)}$. The derivatives at $\Lambda=\hat{\Lambda}$ of $\left|M_{(1)}(\Lambda)\right|$ are:

a) $\partial\left|M_{(i)}(\Lambda)\right| / \partial \lambda_{r}=2\left|Z_{i r}^{\prime}\left(W_{i r}\right) A_{i} Z_{j}\right|$;

b) $\partial^{2}\left|M_{(j)}(\Lambda)\right| / \partial \lambda_{r}^{2}=2\left[\left|Z_{i r}^{\prime}\left(U_{i r}\right) A_{i} Z_{i}\right|+\left|Z_{i r}^{\prime}\left(W_{i r}\right) A_{i} Z_{i r}\left(W_{i r}\right)\right|\right]_{;}$

c) $\partial^{2}\left|M_{(i)}(\Lambda)\right| / \partial \lambda_{s} \partial \lambda_{r}=2\left[\left|Z_{i s r}^{\prime}\left(W_{i s}, W_{i r}\right) A_{i} Z_{i}\right|+\left|Z_{i r}^{\prime}\left(W_{i r}\right) A_{i} Z_{i s}\left(W_{i s}\right)\right|\right](r \neq s)$.

B.2 is based on the following proposition which is stated without proof.

Proposition B.3. For every $i=1, \ldots, n$, we can write

$$
M_{(1)}(\Lambda)=Z_{1}^{(\Lambda)^{\prime}} A_{i} Z_{1}^{(\Lambda)}
$$


C ssymptatic properties of $\hat{\Lambda}_{R}$. If $E_{F}\left[\left|\log X_{j}\right|<\infty\right.$, and $\theta(\Lambda)$ solves $\min |\Sigma|$ over $\theta=(\mu, \Sigma) \quad(\Sigma>0)$ restricted to $\int \rho\left[\left\{\left(x^{(\Lambda)}-\mu\right)^{\prime} \Sigma^{-1}\left(x^{(\Lambda)}-\mu\right)\right\}^{1 / 2}\right] F(d x)=k_{p}$, it can be shown that if $\left\{G_{k}\right\}$ converges weakly to $F$, there exists for all $k$ large enough, a sequence $\left\{\theta\left(\Lambda, G_{k}\right)\right\}$, equicontinuous on $\Lambda \in \mathbb{K}$, which converge pointwise to $\theta(\Lambda)$. Convergence is then uniform. Application to the sequence of empirical cdf's $\left\langle\mathrm{F}_{n}\right\}$ yields strong consistency of $\left\{\hat{\Lambda}_{R}\right\}$. Differentiation under the integral sign holds if $\rho[$.$] is such that the second derivatives of$ the array $\psi$ exist with respect to the parameters and, for every $j$ and $k$, the moment $E_{F}\left[X_{k}^{-8 b}\left|\log X_{k}\right|^{8} X_{j}^{-8 b}\left|\log X_{j}\right|^{8}\right]<\infty$, where $b$ is a positive constant such that $-b \leq \lambda_{j} \leq b$, for $j=1, \ldots, p$. This condition also suffices for the influence function to exist and for the Huber's (1967) conditions to hold.

D Gomputational aspects. Array $\dot{\psi}$ has components

$$
\begin{aligned}
& \dot{\psi}_{1, r, j}=\partial \psi_{1, r} / \partial \lambda_{j}+\sum_{a=1}^{p}\left(\partial \psi_{1, r} / \partial \mu_{a}\right) \omega_{a, j}+\sum_{\substack{a, b=1 \\
a \leq b}}^{p}\left(\partial \psi_{1, r} / \partial \sigma_{a b}\right) \omega_{a b, j}, \\
& \dot{\psi}_{2, r s, j}=\partial \psi_{2, r s} / \partial \lambda_{j}+\sum_{a=1}^{p}\left(\partial \psi_{2, r s} / \partial \mu_{a}\right) \omega_{a, j}+\sum_{\substack{a, b=1 \\
a \leq b}}^{p}\left(\partial \psi_{2, r s} / \partial \sigma_{a b}\right) \omega_{a b, j}, \quad \text { where } \quad \text { for }
\end{aligned}
$$

example,

$$
\begin{aligned}
& \partial \psi_{1, r} / \partial \lambda_{j}=u^{\prime}[d]\left(\partial d / \partial \lambda_{j}\right)\left(x_{r}\left(\lambda_{r}\right)-\mu_{r}\right)+u[d] \delta_{j r}\left(\partial x_{r}\left(\lambda_{r}\right) / \partial \lambda_{r}\right) \\
& \partial \psi_{1, r} / \partial \mu_{a}=u^{\prime}[d]\left(\partial d / \partial \mu_{a}\right)\left(x_{r}\left(\lambda_{r}\right)-\mu_{r}\right)-u[d] \delta_{r a}, \\
& \partial \psi_{1, r} / \partial \sigma_{a b}=u^{\prime}[d]\left(\partial d / \sigma_{a b}\right)\left(x_{r}\left(\lambda_{r}\right)^{\prime}-\mu_{r}\right) .
\end{aligned}
$$

Ailso: $\quad \partial d^{\prime} \partial \lambda=(1 / d)\left(\partial x_{j}\left(\lambda_{j}\right) / \partial \lambda_{j}\right)\left[e^{\prime} \Sigma^{-1}\left(x^{(\Lambda)}-\mu\right)\right], \quad \partial d / \partial \mu_{a}=(1 / d)\left[e_{a}^{\prime} \Sigma^{-1}\left(\mu-x^{(\Lambda)}\right)\right]$, $\left.\partial \mathrm{d} / \partial \sigma_{\mathrm{ab}}=(1 / 2 \mathrm{~d})\left(\mathrm{x}^{\dagger}-\mu\right)\right)^{\prime}\left(\partial \Sigma^{-1} / \partial \sigma_{\mathrm{ab}}\right)\left(\mathrm{x}^{(\Lambda)}-\mu\right)$, for selected canonical vectors $e_{j}$ and $e_{a}$. On the other hand, $\Gamma_{j}=(1 / 2) \sum_{\substack{r, s=1 \\ r \leq s}}^{p}\left[2 \sigma^{r s}-\delta_{r s} \sigma^{r s}\right] \omega_{r s, j}-\log j ; A$ symbolic differentiation code is useful when handling this expressions.

\section{ACKNOWLEDGEMENTS}

Research partially supported by Grant PB90-0266 (Spain). The author is grateful to P. Delicado for helpful computing assistance and estimulating conversations. 


\section{REFERENCES}

ANDREWS, D.F., GNANADESIKAN, R. \& WARNER, J.L. (1971). Transformations of multivariate data. Biametrics, 27, 825-840.

ATKINSON, A. C. (1985). PLats, Iransformations, and Regression, New York, Oxford University Press.

BOX, G.E.P. \& COX, D.R. (1964). An analysis of transformations. Laurnal of the Royal Statistical Yociety, Ser. B, 26, 211-252.

CARROLL, R.J. (1980). A robust method for testing transformations to achieve approximate normality. Lournal of the Royal Ytatistical Yaciety, Ser. B, $42,71-78$.

CARROLL, R. \& RUPPERT, D. (1988). Iransformations and weighting in regression. New York, Chapman and Hall.

COOK, R.D. \& WANG, P.C. (1983). Transformations and influential cases in regression. Technometrics, 25, 337-345.

FLEMING, W. (1977). Functions of several variables. New York, Springer.

HINKLEY, D.V. \& WANG, S. (1988). More About transformations and influential cases in regression. Jechnometrics, 30, 435-440.

HUBER, P.J. (1967). The behavior of maximum likelihood estimates under nonstandard conditions. Frac. Fifth Berkeley Symp. Math. Stat. Probab. 1, 221-233. Univ. Cailfornia Press.

LOPLHA $\ddot{A}$, H.P. (1989). On the relation between S-estimators and M-estimators of multivariate location and covariance. stnnals of Statistics, 17, 1662-1683.

RUPPERT; D. (1992): Computing S estimators for Regression and Multivariate Location/Dispersion. Gournal of Computational and Graphical Statistics, 3, 253-270.

SEBER, G.A.F. \& WILD, C.J. (1989). Nonlinear Regression. New York: J. Wiley.

TSAI, C.L \& WU, X. (1990). Diagnostics in transformation and weighted regression. Technometrics, 32, 315-322.

VELILLA, S. (1993). A note on the multivariate Box-Cox transformation to normality. Statistics and Probability Letters, 17, 259-263. 


\section{CAPTIONS FOR FIGURES}

Figure 3.1 Data of example 3.1 in logs

Figure 3.2 a) Marginal approximations of Tsai and $W u$ (1990) $\hat{\lambda}_{1 M(1)}$ (cont. line) and $\hat{\lambda}_{2 M(i)}$ (dashed line); b) Marginal likelihood displacements $L_{1}^{1}$ (cont. line) and $\mathrm{LD}_{1}^{2}$ (dashed line); c) Combined marginal likelihood displacement $L D_{1}^{1}+L D_{1}^{2}$

Figure 3.3 a) $L D_{i}$; b) $\tilde{L} D_{i}$

Figure 3.4 a) LDM $_{i}$; b) $\tilde{L D M}_{i}$

Figure 4.1 Curves $A R(r)$ (continuous line) and $A L(r)$ (dashed line) 


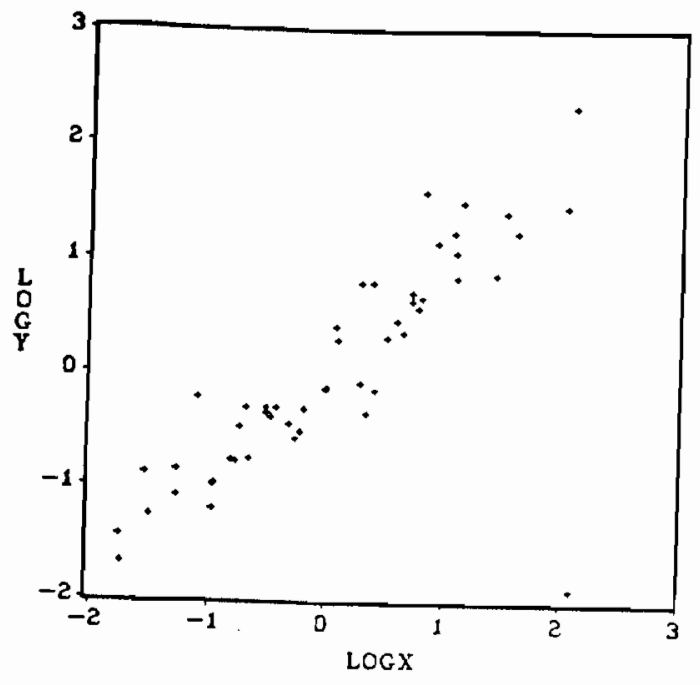

Figure 3.1

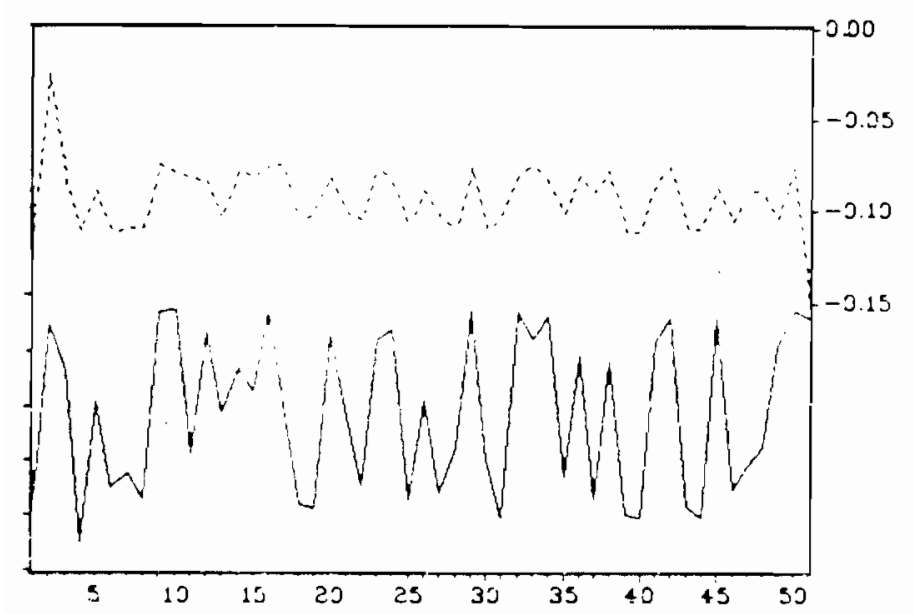

a)

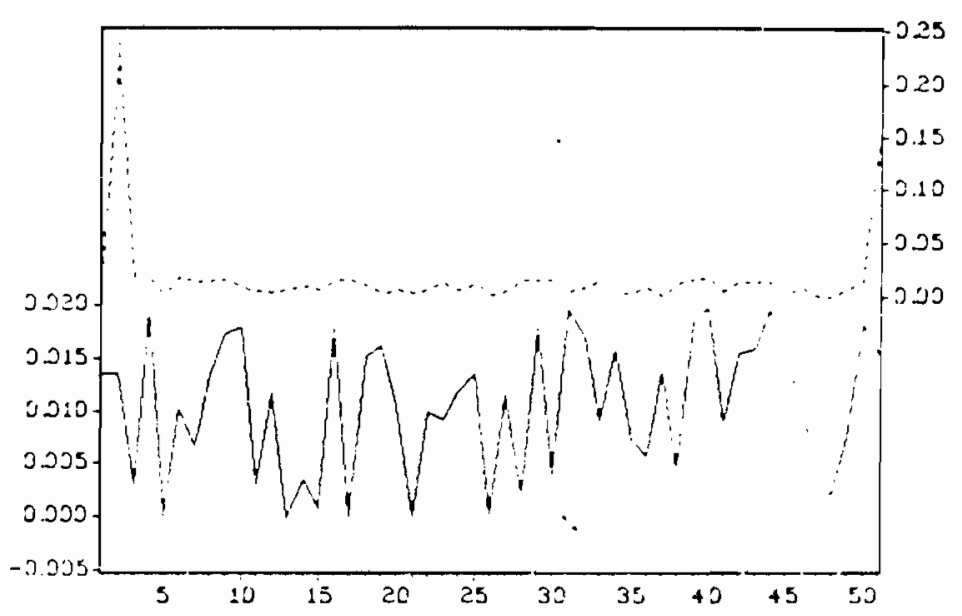

b)

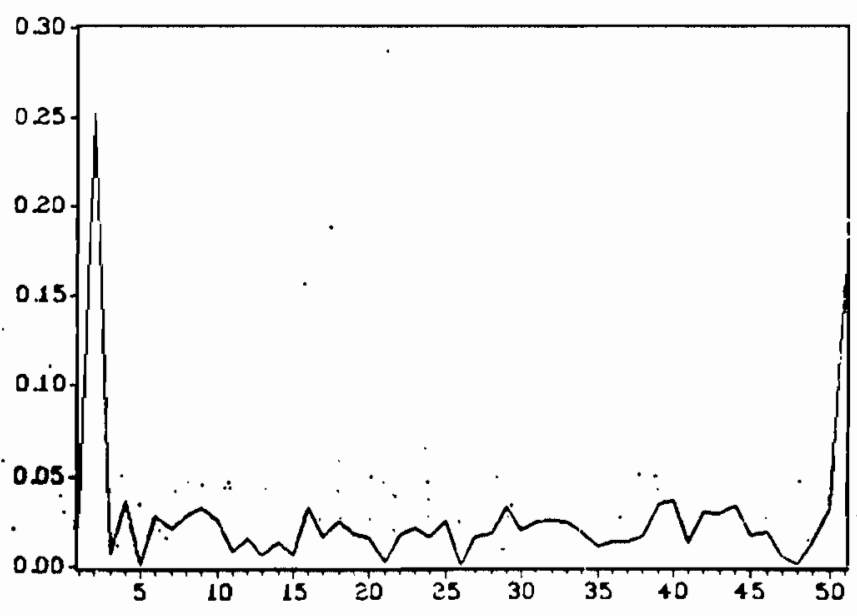

c)

Figure 3.2 


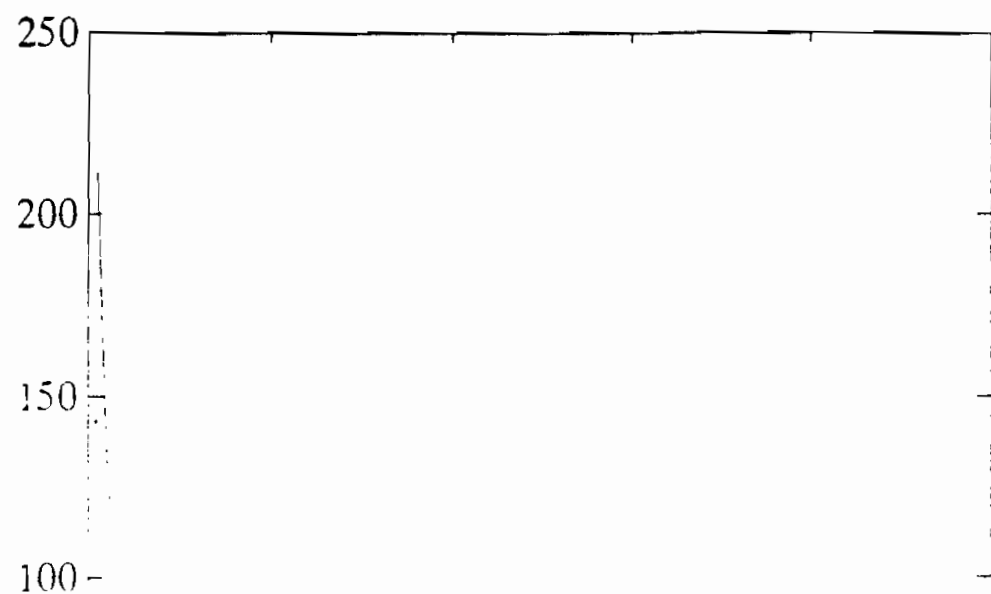

50

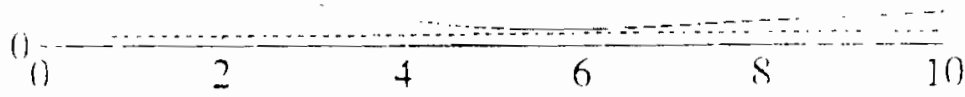

Figure 4.1 